\title{
Méthodologie de l'amélioration de blé tendre (Triticum aestivum L). I. Création par croisements et analyse d'une population artificielle à 16 parents, base de cette étude méthodologique
}

\author{
G Thomas 1, M Rousset 2, M Pichon 2, M Trottet 1, G Doussinault 1, E Picard 3 \\ 1 INRA, station d'amélioration des plantes, domaine de la Motte, BP 29, 35650 Le Rheu; \\ 2 INRA, station d'amélioration des plantes, domaine de Crovelle, 63039 Clermont-Ferrand; \\ ${ }_{3}^{3}$ GIS Moulon, Ferme du moulon, 91190 Gif-sur-Yvette, France
}

(Reçu le 20 mars 1989; accepté le 21 janvier 1991)

\begin{abstract}
Résumé - L'utilisation de la sélection récurrente, généralisée pour les espèces allogames, est peu fréquente chez les espèces autogames du fait des difficultés d'obtention de populations de départ et d'hybridation pour les intercroisements. Pour une étude méthodologique incluant sélection récurrente et création variétale (lignées) chez le blé tendre, une population PA a été réalisée. Elle a pour origine 16 parents lignées pures choisis pour leurs performances propres et leur complémentarité dans une optique d'amélioration large. Sa création résulte d'intercroisements selon un schéma pyramidal équilibré à partir de croisements manuels. Les caractéristiques de cette population, base de départ d'une étude méthodologique présentée ultérieurement, sont analysées et discutées pour de nombreux caractères utiles à l'amélioration du blé. Sur la base des valeurs moyennes et de la variabilité intra-population, cette analyse révèle l'intérêt de la combinaison multiple des lignées parentales et l'efficacité de la série de recombinaisons appliquée dans le schéma pyramidal.
\end{abstract}

population de base / croisement pyramidal / choix des parents / comparaison de méthodes

Summary - Breeding methodology in wheat (Triticum aestivum L). I. Creation and study of a 16-parent artificial population. Recurrent selection, though largely used in outbreeding species, is rarely introduced in self breeding species selection schemes because of initial population production difficulties and of difficult hybridization for intercrossing. For a methodological study including recurrent both selection and pure-line breeding in wheat, an artificial population named PA has been introduced. It comes from 16 parents chosen for their own value and their complementarity for large breeding purposes. It has been produced by manual intercrossing on a pyramidal balanced pattern. The population features are presented and discussed for many characters useful in wheat breeding improvement. This study shows the positive effects of the multiple combination of parents and the efficiency of four recombination cycles introduced in this pyramidal crossing scheme for mean values and intrapopulation variability.

basic population / pyramidal intercrossing / parental choice / selection methods

\section{INTRODUCTION}

L'amélioration de populations par sélection récurrente, largement employée pour la sélection des espèces allogames, présente quelques difficultés de mise en œuvre pour les espèces au- togames. Ces difficultés sont notamment dues à l'absence de populations hétérozygotes à variabilité large comme base de départ et aux difficultés d'intercroisements nécessaires au passage d'un cycle de sélection à l'autre en système récurrent. 
Pour le matériel de départ, la nature et la fréquence initiale des allèles conditionne fortement la probabilité de conserver et de cumuler sur un même génotype les gènes favorables au cours des cycles successifs. C'est pourquoi la base génétique utilisée dans de nombreux schémas est volontairement large. Cette population de départ peut être une "population-source» pour les espèces disposant encore de variabilité “locale». C'est aussi parfois une population créée artificiellement sur le modèle des populations composites de Suneson (1956) (Mak et Harvey, 1982 sur orge; Romero et Frey, 1966 sur avoine). C'est toutefois le plus souvent le modèle de croisements multiples hiérarchisés qui est retenu pour la création de populations. Les parents lignées pures, choisis pour leurs qualités complémentaires, sont en effectif variable selon l'ampleur de la variabilité recherchée (10 parents blé tendre pour Busch et Kofoid, 1982) mais aussi selon les contraintes du plan de croisement $\left(2^{n}\right.$ parents obligatoirement dans le cas d'un plan de croisement pyramidal équilibré; nombre libre dans le cas d'un plan circulaire de croisements (Doussinault et Trottet, 1981).

La création artificielle de ces population requiert la mise en cuvre de méthodes de croisements, inhabituels pour ces espèces adaptées à l'autofécondation. Le recours aux croisements manuels, moyennant un temps de travail important et une technicité fiable, permet d'assurer un intercroisement systématique limitant toutefois l'effectif d'intercroisements (Frey, 1967; Jensen 1978; Burton et Brim 1978). Par ailleurs, le recours à des agents chimiques d'hybridation facilite les intercroisements systématiques avec, actuellement, une fiabilité satisfaisante. Leur usage pour la création d'une base de départ a été envisagé sur triticale (ethephon, Bakinouskaya et al, 1981) et sur orge (Verma et Kumar, 1978). Enfin, l'introduction d'un système de stérilité mâle facilite non seulement la possibilité d'intcicroisements mais aussi l'amplitude du brassage génétique de départ (croisements en plus grand nombre par rapport à la castration manuelle). Sur les principales espèces autogames, les systèmes envisagés sont de type génétique, avec gène dominant de stérilité (Sorrels et Fritz, 1982) mais le plus souvent avec gène récessif de stérilité (Brim et Stuber, 1973 sur soja; Falk et Kasha, 1980 sur orge; Ramage, 1977; Lee, 1984 sur blé).

Le choix raisonné de lignées parentales de base et les modalités d'intercroisement consti- tuent en fait les 2 opérations de base de la création d'une population artificielle pour une espèce autogame.

Dans la présente étude, seront analysées les modalités de création et les caractéristiques d'une population de blé tendre, PA, à partir de 16 parents. Cette population constitue la base de diverses études méthodologiques relatives à l'amélioration génétique de l'espèce (sélection récurrente et amélioration en population; comparaison de méthodes de création variétale).

\section{CRÉATION DE LA POPULATION PA}

Les enjeux méthodologiques fixés dans cette étude et l'objectif général d'amélioration pour l'adaptation aux conditions culturales de la moitié Nord de la France ont conduit à la création d'une population de départ à large variabilité.

La population PA dont les caractéristiques sont précisées ci-après est une population créée artificiellement par croisements pyramidaux successifs à partir de 16 lignées parentales.

\section{Les lignées parentales de PA}

\section{Origine}

Les 16 lignées pures parentales devant satisfaire les objectifs d'amélioration préalablement cités proviennent de 3 groupes différents (tableau I) : variétés commerciales inscrites au Catalogue français; lignées obtenues dans les laboratoires INRA de sélection du blé qui, pour une part (5 lignées), intègrent dans leur généalogie le géniteur de résistance au piétin verse VPM (Maia, 1962) et, pour une autre part (7 lignées), portent les gènes de nanisme de Norin 10 et sont issus de blés sélectionnés au CIMMYT; lignées INRA possédant, dans leur ascendance, des lignées d'origine étrangère.

Globalement ces lignées sont assez apparentées.Cette situation est traduite par le tableau II qui montre les coefficients de parenté entre les lignées fondatrices de la population PA. Dans le choix des lignées, la complémentarité des caractères et le souci d'introduire des allèles favorables à une fréquence élevée ont été privilégiés par rapport à la diversité des origines génétiques. 
Tableau I. Parents de la population PA - Répartition, orignine, généalogie.

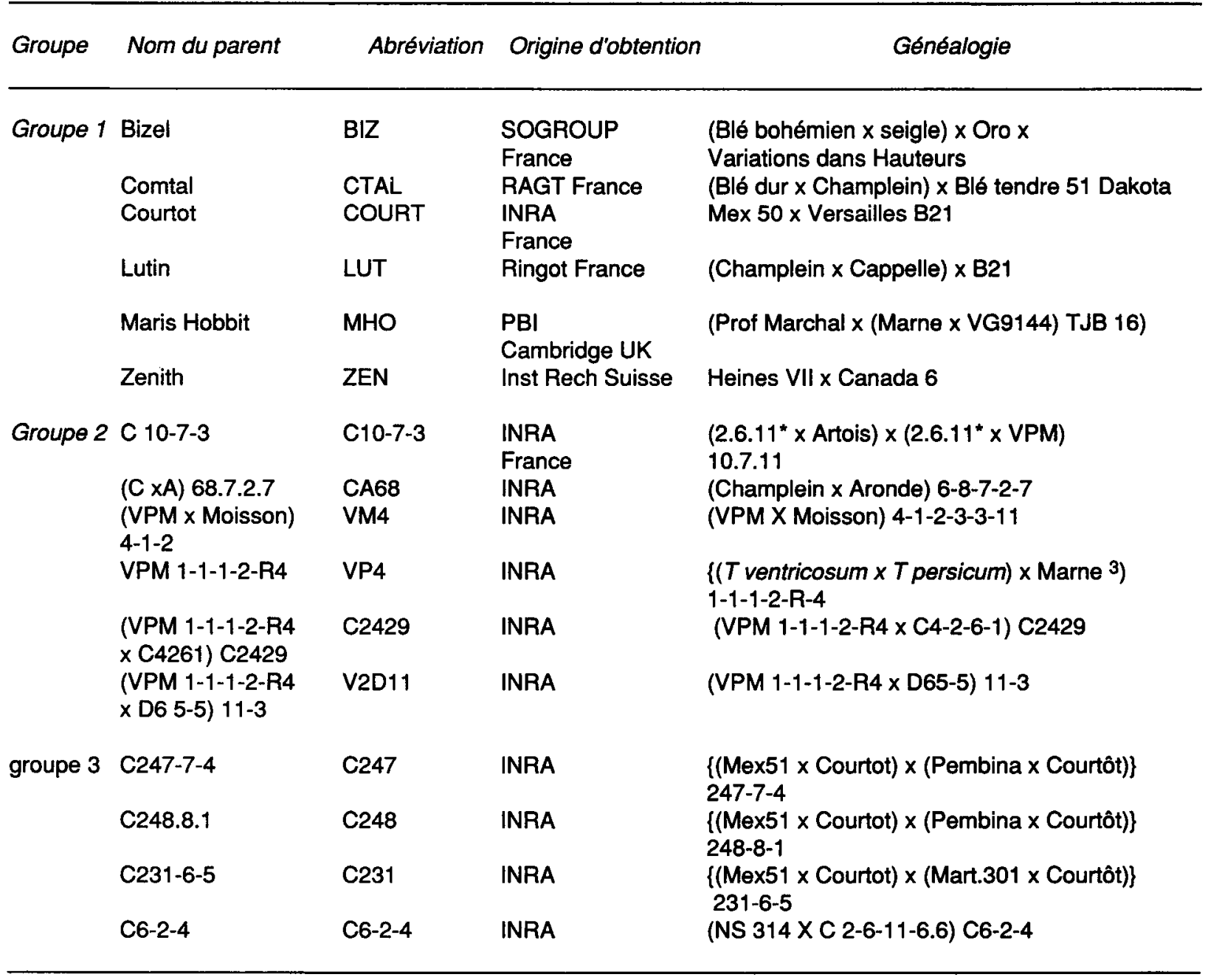

* : 2.6 .11 : Lignée sœur de Courtot

\section{Caractéristiques des lignées parentales}

L'objectif général d'amélioration fixé et les possibilités offertes par la sélection multicaractère ont présidé au choix d'un éventail très varié de lignées rassemblant, de façon connue au préalable, des caractéristiques très diverses.

Les performances des principaux caractères agronomiques et technologiques présentés (tableau III) résultent d'observations et essais sur 7 années à Rennes et permettent de montrer la variabilité existant entre les parents pour un critère donné ainsi que la variabilité de comportement d'un même parent vis-à-vis des caractères retenus.

Globalement, dans le tableau III, la variabilité initiale retenue (valeur propre des 16 parents différents) est décomposée en 3 types de critères.

\section{Productivité et composantes du rendement}

Si plusieurs lignées ont un rendement global de bon niveau (MHO), d'autres présentent seulement un paramètre de productivité élevé (fertilité pour CTAL, poids individuel de grain pour C231,...). Par contre, certaines lignées, performantes par ailleurs, ont une productivité faible (C249, V2D11, Court).

\section{Facteurs de régularité du rendement}

Ces caractères ont fait l'objet d'un choix attentif en essayant d'intégrer :

- la résistance à la verse par l'intermédiaire de la hauteur des plantes (géniteurs de taille courte intégrant les gènes de nanisme rht1, rht2 issus du cultivar Norin 10 (Court, C247, C231, C2429); 


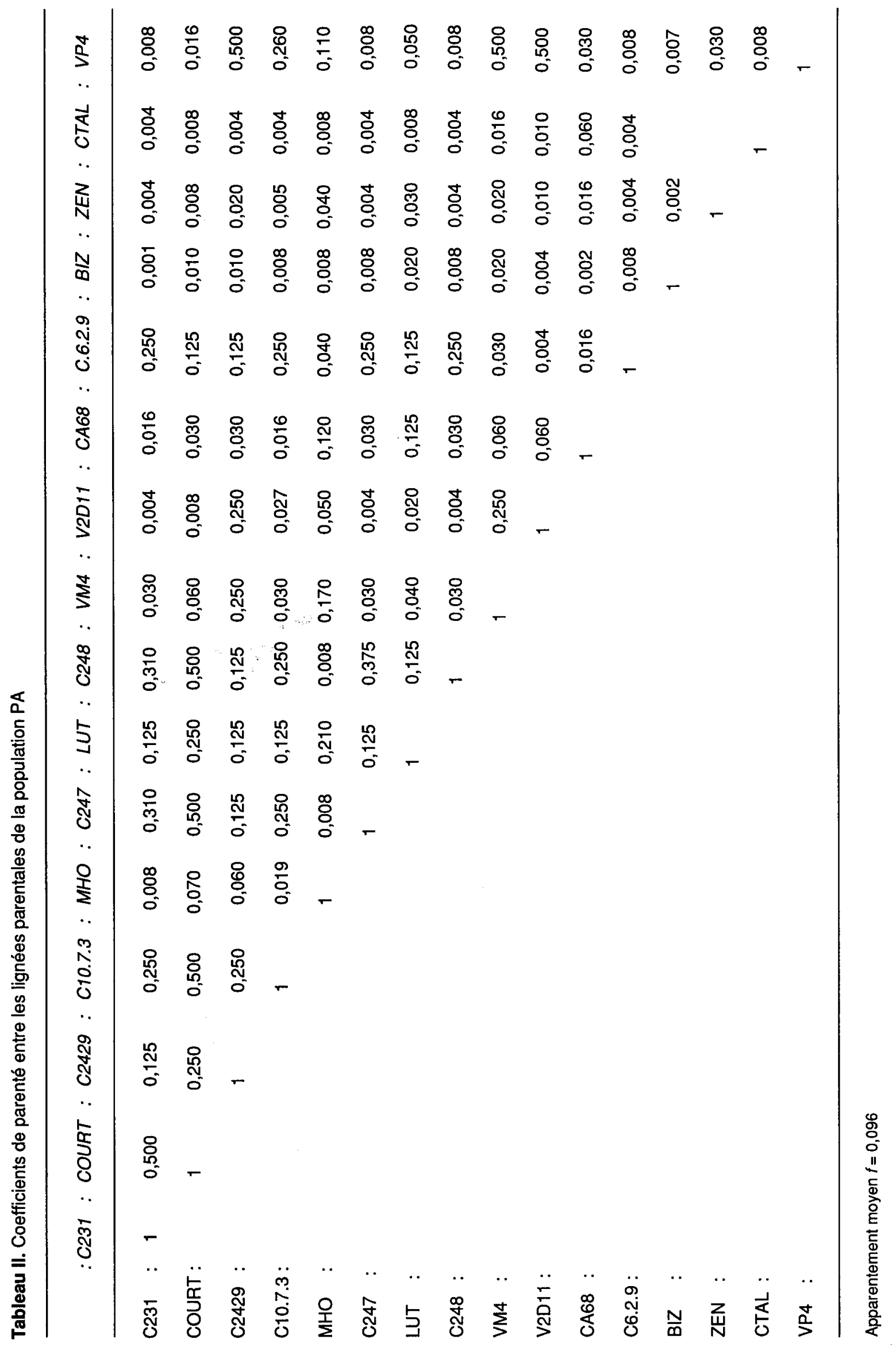


Tableau III. Performances moyennes des 16 parents de la population PAO appréciées sur 7 années d'observation. $E P=$ précocité d'épiaison; OID1 = oïdum feuilles; OID2 = oïdium épis; $R J$ = rouille jaune; $S=$ septoriose; $R N=$ rouille noire; $\mathrm{HA}=$ hauteur; $\mathrm{RC}=$ rapport col/hauteur; $\mathrm{NET}=$ nombre d'épillets; $\mathrm{P3EE}=$ poids de 3 épis entiers (9); P3E = poids de 3 épis battus (9); RDT = rendement $(q /$ ha); PELS = valeur techno (Pelshenke); ZEL = valeur techno (Zeleny). Pour les maladies l'échelle va de 0 à 9 .

\begin{tabular}{lrrll}
\hline Caractère étudié & Moyenne & Ecart type & Parent min & Parent max \\
\hline & & & & \\
EP * & 9,19 & 3,20 & V2D11 $(2,40)$ & MHO $(13,10)$ \\
OID1 & 3,32 & 1,05 & CA68 $(1,80)$ & VP4 $(4,76)$ \\
OID2 & 1,72 & 1,02 & CTALZEN/BIZ $(1,00)$ & COURT $(3,28)$ \\
RJ & 1,74 & 0,80 & C10.7.3 $(1,00)$ & C247 $(3,60)$ \\
RB & 2,36 & 1,34 & VP4 $(1,04)$ & LUT $(4,97)$ \\
S & 2,96 & 0,34 & VP4 $(2,41)$ & LUT $(3,63)$ \\
RN & 1,12 & 0,30 & n $(1,00)$ & MHO $(3,20$ \\
HA & 77,20 & 16,10 & C2429 $(55,40)$ & VP4 $(116,90)$ \\
RC & 0,35 & 0,03 & C2429 (0,28) & Cx A68 $(0,41)$ \\
NET & 20,17 & 1,15 & COURT $(17,86)$ & MHO $(21,43)$ \\
P3EE & 6,07 & 1,24 & BIZ $(4,68)$ & CTAL $(8,88)$ \\
P3E & 4,13 & 1,15 & ZEN $(2,93)$ & VM4 $(6,39)$ \\
NG3E & 123,70 & 30,65 & C2429 (70,60) & CTAL $(212,70)$ \\
PMG & 27,80 & 3,93 & MHO $(22,20)$ & C231 $(34,00)$ \\
RDT & 71,78 & 8,51 & VP4 $(56,30)$ & C231 (89,40) \\
PELS & 103,28 & 59,36 & MHO $(22,00)$ & C248 $(224,00)$ \\
ZEL & 35,00 & 9,06 & CTAL $(20,70)$ & C247 $(53,70)$ \\
\hline
\end{tabular}

Parent $\mathrm{min}$ = indique, pour chaque caractère, le nom du parent ayant la valeur la plus faible avec, cette valeur minimale; parent max = indique, pour chaque caractère, le nom du parent ayant la valeur la plus forte, avec cette valeur maximale; * EP : mesurée en nombre de j par rapport à une date d'origine.

- la résistance aux maladies combinant de nombreux parasites, actuellement importants en culture. Peuvent être citées des lignées présentant un bon niveau de résistance pour les maladies du feuillage (C10.7.3, VP4 pour la rouille jaune; CTAL; V2D11 pour la rouille brune; VP4, VM4, C231 pour la rouille noire). De même, des résistances partielles à la septoriose (CTAL, BIZ, VP4), à l'oïdium (CA68, C247), à la fusariose (BIZ) ont été repérées pour être introduites dans la population. Pour le piétin-verse, la résistance élevée du géniteur VPM provenant d'une action combinée gène-cytoplasme issue de Triticum ventricosum Tausch a été retenue par l'intermédiaire de lignées dérivées de VPM : VP4, V2D11, VM4, C10.7.3.

\section{Qualité technologique}

Les résultats de tests prédicteurs de la valeur technologique ont permis de retenir dans le pool parental, des lignées à fortes potentialités technologiques : C248, Court, C247, C10.7.3 (test Pelshenke supérieur à 150; Zeleny supérieur à 40).

\section{Liaisons entre caractères}

Compte-tenu du nombre important de caractères retenus pour le choix des parents, les 16 lignées ne sont généralement pas satisfaisantes pour l'ensemble des critères retenus. On pourra ainsi noter les antagonismes suivants :

- lignées de hauteur faible mais sensibles à certains parasites (rouilles) : Court, C247;

- lignées tolérantes à la septoriose mais de taille élevée : Ctal, Zen, Biz,VP4;

- lignées résistantes aux rouilles et au piétinverse mais hautes, sensibles à l'oïdium : VP4, V2D11;

- lignées à fort rendement mais de qualité technologique médiocre : $\mathrm{MHO}$

La généralisation partielle à l'ensemble des 16 parents utilisés peut être faite à l'examen des corrélations entre caractères pour l'ensemble des parents (tableau IV). On signalera notamment les liaisons négatives : oïdium/ rouille brune, oïdium/hauteur, septoriose/ hauteur, représentatives d'une sensibilité accrue pour les génotypes de taille courte. Les 


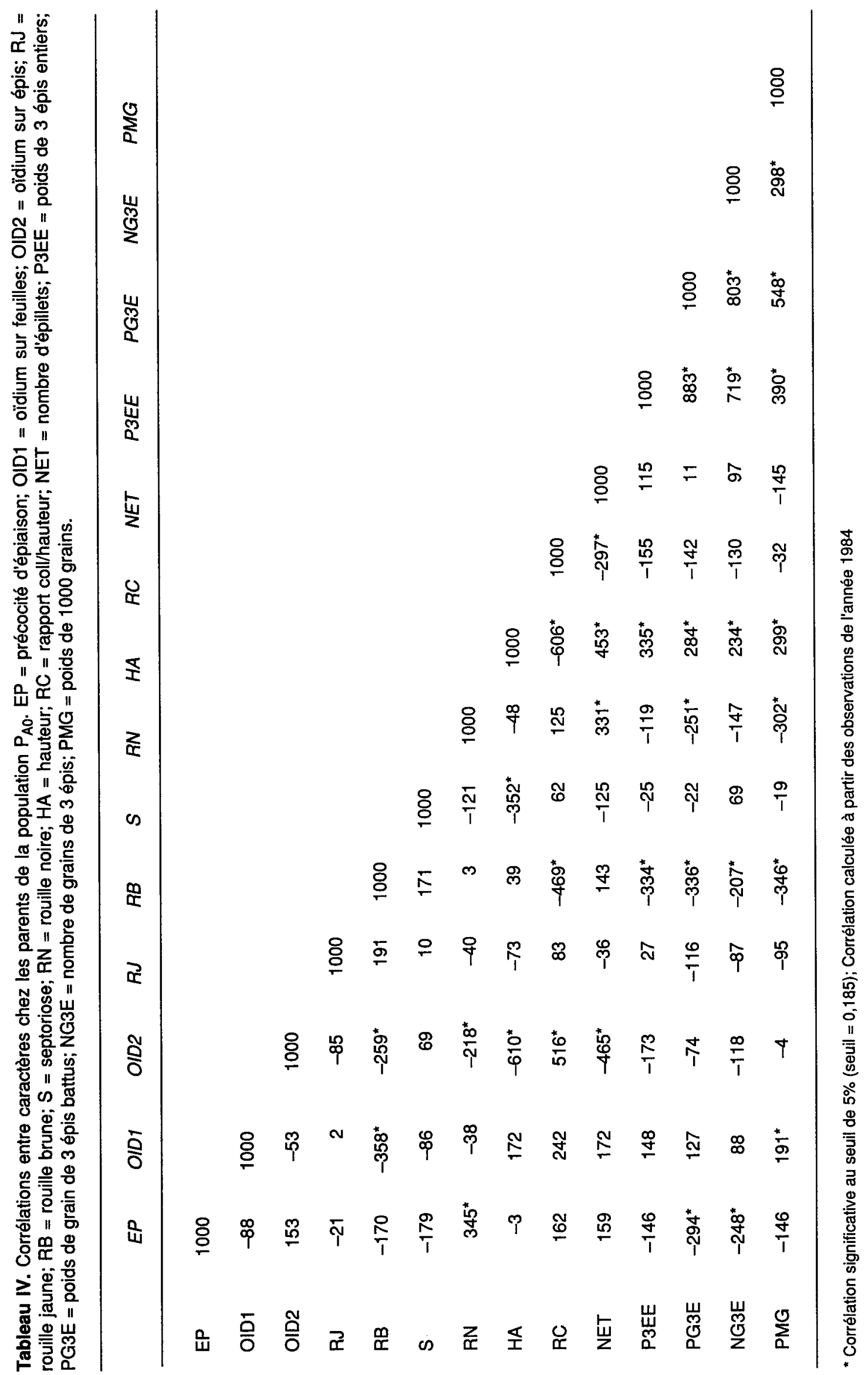


autres corrélations, notamment entre paramètres du rendement, sont, quant à elles, classiques dans l'espèce.

Globalement, les lignées retenues sont donc performantes au moins dans une des catégories d'objectifs fixés (rendement, régularité, qualité). Même si elles présentent certains "défauts", les lacunes de chacune sont systématiquement équilibrées par des niveaux élevés pour d'autres caractères, justifiant ainsi leur choix dans le pool de base ayant servi à la réalisation de la population initiale PA.

\section{Modalités de création de la population PA}

\section{Le plan de croisement}

L'option choisie étant la création d'une population à base large, le nombre élevé de lignées initiales retenues permet diverses modalités d'intercroisements. Le premier niveau de choix est représenté par le mode d'interpollinisation, ici effectué par castration manuelle.Ce système plus lourd, mais aussi plus précis, de mise en œuvre a été choisi pour garder notamment toutes les informations généalogiques structurées et la hiérarchie des croisements. Limité à des appariements par couples de plantes, il est toutefois plus restrictif pour le brassage génétique que l'introduction et l'usage d'un système de stérilité mâle, utilisable dans l'espèce.
Au second niveau de choix, dans ce système manuel, diverses modalités de combinaisons des lignées sont envisageables parmi lesquelles a été retenu le plan pyramidal de croisements. Classiquement, ce dispositif fait intervenir chacun des parents une seule fois, comme mâle ou comme femelle. Avec un effectif initial de 16 lignées $\left(2^{4}\right)$, ce schéma permet d'introduire 4 méioses avec recombinaisons efficaces. De plus, chaque individu contient, en probabilité, une proportion égale du génotype de chaque parent.

\section{Modalités de croisements}

Le plan pyramidal appliqué aux 16 parents, échelonné sur 4 années de croisements, est présenté à la figure 1 .

Pour les 2 premières générations de croisements, chaque génotype parental intervient une seule fois, soit comme mâle, soit comme femelle, comme dans le dispositf classique, mais pour les 2 dernières générations, (création des hybrides à 8 et 16 parents), chaque type de génotype hybride intervient à la fois comme mâle et comme femelle. À ce stade, chaque plante considérée comme femelle est castrée et pollinisée par 3 inflorescences provenant de 3 plantes tirées au sort dans le lot de plantes appartenant au "génotype" pris comme mâle. Susceptible d'améliorer le brassage génétique,

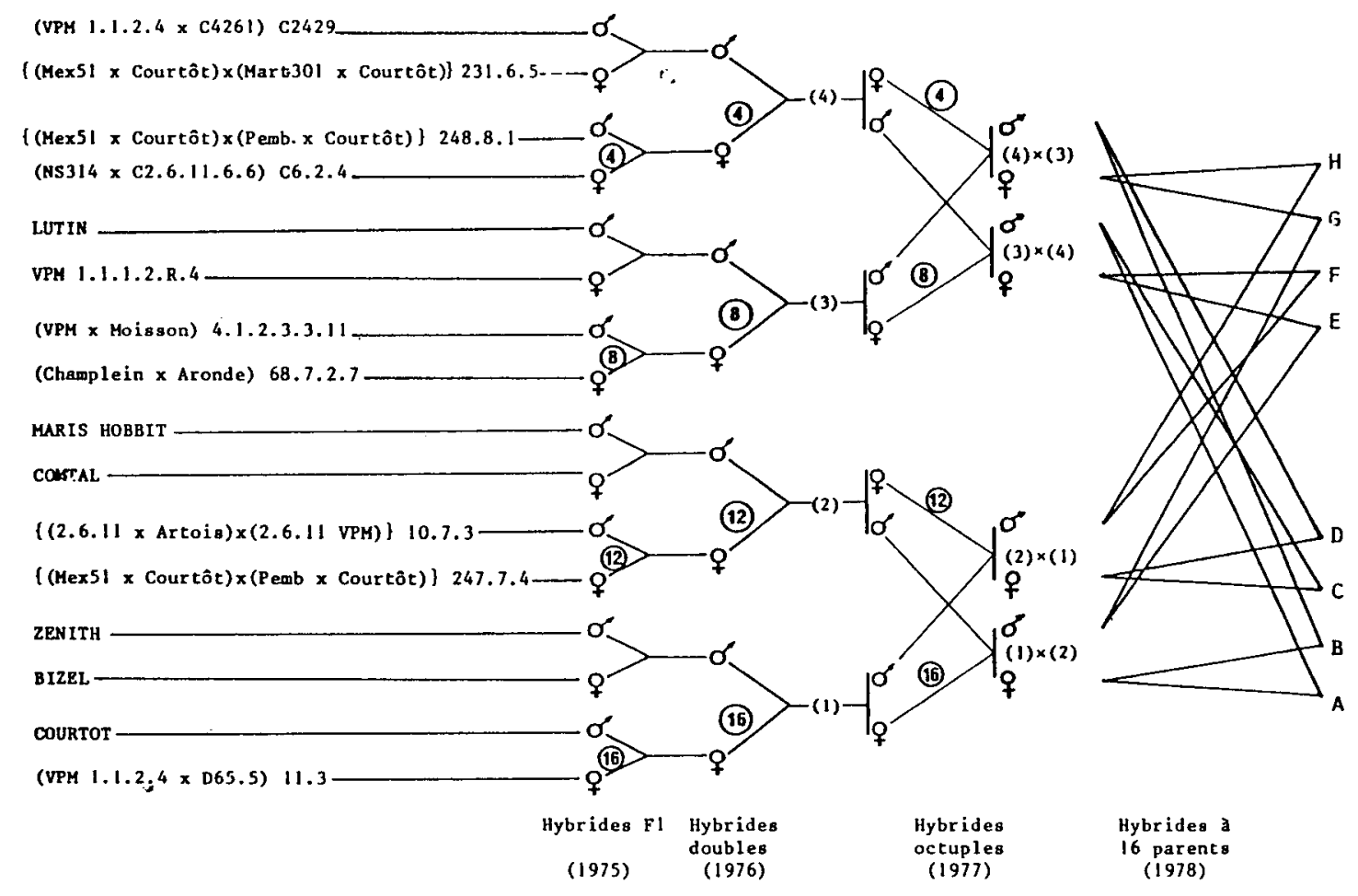

Fig 1. Schéma de création de la population de départ PAo. 
cette pollinisation multiple conduit toutefois à une variation généalogique pour les descendants d'une même plante femelle.

Le bilan d'obtention des croisements figure au tableau V. II fait apparaître la production après 4 générations d'un ensemble d'hybrides à 16 parents, répartis en 8 groupes d'effectifs équivalents, reflétant 8 types de croisements pyramidaux. Cet ensemble de 8 origines (répertoriées de $\mathrm{A}$ à $\mathrm{H}$ ) constitue la population artificielle PA, base de notre étude méthodologique.

\section{ANALYSE DES CARACTÉRISTIQUES DE LA POPULATION PA}

La population $\mathrm{PA}_{0}$ représente la population initiale, base de l'étude méthodologique précisée ci-dessus. Nous nous attacherons ici à analyser uniquement la population $\mathrm{PA}_{0}$ par rapport à ses parents de départ, notamment pour présenter l'état initial de cet outil d'expérimentation.

\section{Mode d'analyse}

La population $\mathrm{PA}_{0}$ issue du système de croisements pyramidaux a été analysée comparativement à une population fictive $P_{0}$ constituée de l'ensemble des 16 lignées parentales étudiées en parallèle. Cette étude a été conduite à Rennes, en pépinière, durant 2 années consécutives. La population $\mathrm{PA}_{0}$, répartie en 8 origines, a été observée sur la base de familles, chaque famille représentant la descendance d'une plante hybride octuple pollinisée par 3 plantes mâles. Chaque famille est étudiée à raison de 11 plantes individualisées sur une ligne de $1,50 \mathrm{~m}$. Les 16 parents, à titre de référence, ont été insérés régulièrement dans cette pépinière $P A_{0}$, en

Tableau V. Bilan de réalisation des croisements de $\mathrm{PA}_{0}$

\begin{tabular}{|c|c|c|c|}
\hline Année & Type de croisement & $\begin{array}{l}\text { Effectif par croisement } \\
\text { par station }\end{array}$ & $\begin{array}{c}\text { Effectif total de } \\
\text { croisement }\end{array}$ \\
\hline 1975 & $\begin{array}{l}\text { V2D } 11 \times \text { COURT } \\
\text { C247 } x=\text { C10.7.3 } \\
\text { BIZ } \times \text { ZEN } \\
\text { CA68 } \times \text { VM4 } \\
\text { CTAL } \times \text { MHO } \\
\text { VP } 4 \times \text { LUT } \\
\text { C624 x C248 } \\
\text { C231 } \times \text { C2429 }\end{array}$ & $\begin{array}{l}1 \\
1 \\
1 \\
1 \\
1 \\
1 \\
1 \\
1\end{array}$ & 16 \\
\hline 1976 & $\begin{array}{l}(\text { V2D11 } \times \text { COURT }) \times(B I Z \times \text { ZEN }):(1) \\
(\text { C247 } \times \text { C10.7.3) } \times(\text { COURT } \times \text { MHO }):(2) \\
(\text { CA68 } \times \text { VM4 }) \times(\text { VP } 4 \times \text { LUT }):(3) \\
(\text { C624 } \times \text { C248) } \times(\text { C231 } \times \text { C2429) }:(4)\end{array}$ & $\begin{array}{l}4 \\
12 \\
77 \\
27\end{array}$ & 120 \\
\hline 1977 & $\begin{array}{l}\text { (1) } \times(2) \\
(2) \times(1) \\
(3) \times(4) \\
(4) \times(3)\end{array}$ & $\begin{array}{l}12 \\
12 \\
12 \\
12\end{array}$ & 96 \\
\hline 1978 & $\begin{array}{l}\{(1) \times(2)\} \times\{(3) \times(4)\} \\
\{(2) \times(1)\} \times\{(3) \times(4)\} \\
\{(1) \times(2)\} \times\{(4) \times(3)\} \\
\{(2) \times(1)\} \times\{(4) \times(3)\} \\
\{(4) \times(3)\} \times\{(1) \times(2)\} \\
\{(4) \times(3)\} \times\{(2) \times(1)\} \\
\{(3) \times(4)\} \times\{(1) \times(2)\} \\
\{(3) \times(4)\} \times\{(2) \times(1)\}\end{array}$ & $\begin{array}{l}25 \\
25 \\
25 \\
25 \\
25 \\
25 \\
25 \\
25\end{array}$ & 200 \\
\hline 1979 & & $\begin{array}{c}1551 \text { plantes } \mathrm{PA}_{0} \\
\text { observées }\end{array}$ & \\
\hline
\end{tabular}


bloc de 16 lignes de 11 plantes sur $1,50 \mathrm{~m}$, toutes les 25 familles. L'étude intègre l'analyse des performances moyennes de ces 2 populations ainsi que celle des potentialités extrêmes (génotypes de valeur maximale et minimale pour chaque caractère). Les caractères étudiés sont ceux qui sont ensuite analysés dans le schéma de sélection récurrente. L'étude comparative porte donc sur l'évolution de la population par rapport à ses géniteurs de départ, permettant ainsi de caractériser les effets du système de croisements pyramidaux.

\section{RÉSULTATS}

\section{Performances moyennes}

Sur l'ensemble des 2 années d'étude (tableau $\mathrm{VI})$, les performances de la population $\mathrm{PA}_{0}$ sont supérieures (dans le sens positif de l'amélioration) pour un certain nombre de caractères : résistance aux maladies (oïdium, piétin-verse), paramètres du rendement (poids d'épi, poids de grain, nombre de grains). Par contre, certains caractères voient leur niveau moyen baisser entre $P_{0}$ et la création de $P A_{0}$. Ceci concerne certaines résistances aux maladies (oïdium, rouilles, septoriose) la hauteur des plantes, la fertilité (nombre d'épillets), le poids de 1000 grains.
On observe donc au cours de cette création de populations par intercroisements, un véritable remaniement génétique par brassage conduisant, au moins dans la population d'ensemble, à une modification des niveaux. Les 4 méioses efficaces ont donc induit une restructuration partielle qui conduit à une nouvelle situation génétique très hétérozygote qui n'est pas toujours favorable. Cette première approche globale sous l'angle des moyennes est toutefois insuffisante et doit être complétée par une analyse des niveaux extrêmes des caractères dans la population.

\section{Niveaux extrêmes}

Cette étude, faite uniquement sur une année (année 1983, tableau VI) a été réalisée en comparant la moyenne des différentes familles $P_{0}$ à la moyenne de chaque parent. Elle ne tient donc pas compte de la variation intrafamille qui reste importante.

Globalement, on note un accroissement de variabilité pour tous les caractères mesurés. Le niveau le plus favorable du caractère présent chez les parents est atteint dans le cas de la résistance aux maladies. Il dépasse celui du meilleur parent pour certains facteurs du rendement, comme le nombre d'épillets et le poids de grain de 3 épis. Par contre, il ne l'atteint pas

Tableau VI. Analyse des moyennes de $\left(\mathrm{PA}_{0}\right)$ par rapport aux parents de départ $\left(\mathrm{P}_{0}\right)$ (valeurs moyennes et limites); pour la signification des abréviations des caractères étudiés, voir tableau IV.

\begin{tabular}{|c|c|c|c|c|c|c|c|c|}
\hline \multirow{2}{*}{$\begin{array}{l}\text { Caractères } \\
\text { étudiés }\end{array}$} & \multicolumn{6}{|c|}{1983} & \multicolumn{2}{|c|}{1982} \\
\hline & Moy $P_{0}$ & Moy $P A_{0}$ & $\operatorname{Max} P_{0}$ & $\operatorname{Max} P A_{O}$ & $\operatorname{Min} P_{0}$ & $M i n P A_{O}$ & Moy $P A_{0}$ & Moy $P_{0}$ \\
\hline OID1 (1-9) & 3,10 & 3,46 & 4,95 & 5,60 & 1,35 & 1,40 & 3,44 & 3,32 \\
\hline OID2 (1-9) & 1,72 & & 3,28 & & 1,00 & & & \\
\hline RJ (1-9) & 1,09 & & 2,50 & & 1,00 & 1,00 & 2,62 & 1,53 \\
\hline RB (1-9) & 1,62 & 1,81 & 5,08 & 5,80 & 1,00 & 1,00 & 2,98 & 2,14 \\
\hline$S(1-9)$ & 2,96 & 3,64 & 3,63 & & 2,41 & 2,50 & & \\
\hline $\operatorname{RN}(1-9)$ & 1,24 & 3,64 & 3,19 & 7,00 & 1,00 & 1,00 & & \\
\hline $\mathrm{HA}(\mathrm{cm})$ & 73,96 & 73,34 & 106,21 & 102,72 & 54,08 & 49,70 & 96,15 & 70,13 \\
\hline $\mathrm{RC}$ & 0,16 & 0,14 & 0,23 & 0,23 & 0,10 & 0,06 & & \\
\hline NET & 20,17 & 19,17 & 21,43 & 23,00 & 17,86 & 15,50 & & \\
\hline P3EE (9) & 6,07 & 7,02 & 8,88 & 9,99 & 4,68 & 3,96 & & \\
\hline PG3E & 3,74 & 4,31 & 6,73 & 8,63 & 2,36 & 1,71 & 4,70 & 4,55 \\
\hline NG3E & 123,72 & 133,00 & 212,70 & 204,00 & 70,60 & 49,30 & & \\
\hline PMG & 27,80 & 21,20 & 33,90 & 28,30 & 22,10 & 7,30 & & \\
\hline
\end{tabular}

La valeur «maximale" et «minimum» inscrite représente la valeur de la famille la plus ou la moins performante dans la population étudiée $\{$ PAO ou PO (parents)\}. 
pour le nombre de grains dans 3 épis et le poids de 1000 grains.

L'analyse comparative de $\mathrm{PA}_{0}$ et $\mathrm{P}_{0}$ confirme l'effet attendu du système de croisements pour 4 générations en pyramide, sur l'élargissement de la variabilité observée. La série de 4 méioses efficaces, conséquence du schéma d'intercroisements retenu, modifie totalement la situation génétique initiale. Les recombinaisons ont eu par exemple pour conséquence, une diminution de la fréquence des familles résistantes aux rouilles par rapport à celles des parents. Mais cette constation traduit une probable diversité (révélée par l'intercroisement) au niveau des gènes et des allèles de résistance, tout en permettant probablement l'accumulation de facteur de résistance dans certains génotypes qu'il conviendrait de repérer. Par contre, pour certains caractères à hérédité quantitative, l'accumulation de caractères favorables s'est traduite par une amélioration importante de certains facteurs du rendement comme le nombre d'épillets et le poids de grain de 3 épis pour les meilleures familles.

Cette augmentation de la variabilité pour un caractère s'accompagne aussi d'une redistribution originale de l'ensemble des caractères dans les différentes familles par rapport aux parents.

Bien qu'ayant un apparentement assez important, les parents ainsi combinés ont permis de créer une population montrant une grande variabilité. La série de 4 intercroisements a également provoqué des recombinaisons intéressantes révélées par association de caractères favorables.

Cette population artificielle, de par sa diversité, répond ainsi aux besoins des analyses méthodologiques orientées vers l'étude et la mise en cuvre d'un programme de sélection récurrente à cycle court.

Les résultats méthodologiques relatifs à cette étude entreprise conjointement par les 3 laboratoires INRA d'amélioration du blé de Rennes, Clermont-Ferrand et le GIS Moulon feront l'objet de publications ultérieures où la présentation de la population de base PA ne sera pas reprise.

\section{RÉFÉRENCES}

Bakinouskaya EA, Kaminskaya LM, Khatyleva LU (1981) Use of ethrel in the recurrent selection of Triticale. Ref Zhurnal 3, 169

Brim CA, Stuber CW (1973) Application of genetic male sterility to recurrent selection schemes in soybeans. Crop Sci 13, 528-530

Burton GW, Brim CA (1978) Recurrent mass and within half-sib family selection for high percent oil in soybeans. Crop Sci 18, 320-323

Busch RH, Kofoid K (1982) Recurrent selection for kernel weight in spring wheat. Crop Sci 22, 568-572

Doussinault G, Trottet M (1981) Application de la sélection récurrente aux espèces autogames ou allogames non strictes : allogamisation des autogames. Sel Fr 29, 25-33

Falk DE, Kasha KJ (1980) A shrunken endosperm (sex 1), male sterile (msg6) stock to facilitate crossing in barley. Barley Gen News/ 10, 81-83

Frey KJ (1967) Mass selection for seed width in oat populations. Euphytica 16, 341-349

Jensen NF (1978) Composite breeding methods and the DSM system in cereals. Crop Sci 7, 43-46

Lee BH (1984) Efficiency of male sterile facilitated recurrent selection for earliness in wheat breeding. Res Rep Off Rural Dev 26, 61-77

Maia N (1962) Obtention de blés tendres résistants au Piétin-verse (Cercosporella herpotrichoides) par croisements interspécifiques. CR Séances Acad Agric Fr 53, 149-154

Mak C, Harvey BL (1982) Exploitable genetic variation in a composite bulk population of barley. Euphytica 31, 85-92

Ramage RT (1977) Varietal improvement of wheat through male sterile facilitated recurrent selection. Tech Bull, ASPAC Food Fertil Technol Center, $n^{\circ}$ 37, $6 \mathrm{p}$

Romero GE, Frey KJ (1966) Mass selection for plant height in oat populations. Crop Sci 6, 283-287

Sorrels ME, Fritz SE (1982) Applications of a dominant male-sterile allele to the improvement of selfpollinated crops. Crop Sci 22, 1033-1035

Suneson CA (1956) An evolutionary plant breeding method. Agron J 48, 188-191

Verma MM, Kumar J (1978) Ethrel - a male gametocide that can replace the male sterility genes in barley. Euphytica 27, 865-868 